Proyecciones Journal of Mathematics Vol. 32, No 1, pp. 1-13, March 2013.

Universidad Católica del Norte

Antofagasta - Chile

\title{
A geometric proof of the Lelong-Poincaré formula
}

\author{
M. El Amrani \\ Université d'Angers, France \\ and \\ A. Jeddi \\ Université (et Archives de) H. Poincaré, France
}

Received : October 2012. Accepted : November 2012

\begin{abstract}
We propose a geometric proof of the fundamental Lelong-Poincaré formula : $d d^{c} \log |f|=[f=0]$ where $f$ is any nonzero holomorphic function defined on a complex analytic manifold $V$ and $[f=0]$ is the integration current on the divisor of the zeroes of $f$.

Our approach is based, via the local parametrization theorem, on a precise study of the local geometry of the hypersurface given by $f$. Our proof extends naturally to the meromorphic case.
\end{abstract}

Keywords : Complex analytic manifolds, analytic sets, local parametrization theorem; integration currents, branching coverings.

AMS Subject Class. (2010) : 32B10, 32C30, 32U40. 
Since the Lelong-Poincaré formula plays a crucial role in complex analytic geometry, notably in intersection theory (see [4]), it is a natural aim to look for a geometric proof of this fundamental formula. More precisely, we offer a geometric proof of the following

Theorem Let $V$ be a connected complex analytic manifold of dimension $n$ and let $f: V \rightarrow \mathbf{C}$ be a holomorphic nonzero function. Then, the meromorphic differential form $d^{\prime} f / f$ defines a current of type $(1,0)$ on $V$, and furthermore, we have

$$
d^{\prime \prime}\left[\frac{1}{2 i \pi} \frac{d^{\prime} f}{f}\right]=[f=0]
$$

where $[f=0]$ is the integration current on the divisor of the zeroes of $f$.

We denote by $\mathcal{D}(V)$ the set of compactly supported differential forms of class $C^{\infty}$ in $V$.

Recall that if $T$ is a current of degree $s$ on $V$, then $d T$ is the current of degree $s+1$ acting by the rule :

$$
d T(\varphi)=\langle d T, \varphi\rangle:=(-1)^{s+1}\langle T, d \varphi\rangle=(-1)^{s+1} T(d \varphi), \quad \varphi \in \mathcal{D}(V),
$$

with $d=d^{\prime}+d^{\prime \prime}$ where $d^{\prime}$ and $d^{\prime \prime}$ are holomorphic and antiholomorphic differentiation operator, respectively. So, for any $(n-1, n-1)$ - form $\varphi$ in $\mathcal{D}(V)$, we have

$$
\begin{gathered}
d\left[\frac{1}{2 i \pi} \frac{d f}{f}\right](\varphi)=d\left[\frac{1}{2 i \pi} \frac{d^{\prime} f}{f}\right](\varphi)=\left[\frac{1}{2 i \pi} \frac{d^{\prime} f}{f}\right](d \varphi) \\
=\left[\frac{1}{2 i \pi} \frac{d^{\prime} f}{f}\right]\left(d^{\prime \prime} \varphi\right)=d^{\prime \prime}\left[\frac{1}{2 i \pi} \frac{d^{\prime} f}{f}\right](\varphi) .
\end{gathered}
$$

Recall also that the integration current exists on any analytic set (see $[6])$, and if $\omega$ is a locally integrable $(p, q)$ - form on $V$, it defines a current $[\omega]$ of type $(p, q)$ on $V$ by the formula:

$$
[\omega](\varphi):=\langle[\omega], \varphi\rangle=\int_{V} \omega \wedge \varphi,
$$


where $\varphi$ is any $(n-p, n-q)$ - form in $\mathcal{D}(V)$.

Now, here is the outline of our proof.

By an argument of partition of unity, we easily see that our problem is local on $V$, and then we may assume that $V$ is a domain (open and connected set) of $\mathbf{C}^{n}$ containing 0 , such that $f(0)=0$ and $f$ is nonzero on $V$.

The proof of the theorem can be divided into five steps :

- Existence of the current $\left[d^{\prime} f / f\right]$ when $V=\mathbf{C}$ and $f=P \in \mathbf{C}[z]$.

- Proof of $(L P)$ when $V=\mathbf{C}$ and $f=P \in \mathbf{C}[z]$.

- Existence of the current $\left[d^{\prime} f / f\right]$ in the general case.

- Proof of $(L P)$ for a special class of test forms.

- Proof of $(L P)$ in the general case.

The two firs steps are quite elementary applications from analysis in one complex variable. We focus now on the three last steps.

\section{$\S 1$. Existence of the current $\left[d^{\prime} f / f\right]$ in the general case.}

As $f$ is nonzero on $V$, we can choose a local coordinates system $\left(z_{1}, \ldots, z_{n}\right)$ such that, for any $j \in\{1, \ldots, n\}$, the partial function $\xi \mapsto$ $f(0, \ldots, \xi, \ldots, 0)$, obtained by varying $z_{j}$, is nonzero in a neighborhood of the origin.

Then we have

$$
\frac{d^{\prime} f\left(z_{1}, \ldots, z_{n}\right)}{f\left(z_{1}, \ldots, z_{n}\right)}=\sum_{j=1}^{n} \frac{1}{f\left(z_{1}, \ldots, z_{n}\right)} \frac{\partial f\left(z_{1}, \ldots, z_{n}\right)}{\partial z_{j}} d z_{j},
$$

and we are going to see that the coefficient of $d z_{n}$ is locally integrable on $V$. The proof is obviously analogous for the coefficients of $d z_{j}$ when $1 \leq j \leq n-1$. 
By the Weierstrass preparation theorem, and up to a restriction of the open set $V$, we may assume that $V=\Omega \times D(0, \varepsilon), \varepsilon>0$, where $\Omega$ is a domain in $\mathbf{C}^{n-1}$ containing 0 , and where $f$ can be written, by setting $t=\left(z_{1}, \ldots, z_{n-1}\right)$ and $z=z_{n}$ :

$$
f(t, z)=I(t, z) P_{t}(z), \quad(t, z) \in \Omega \times D(0, \varepsilon),
$$

where $I$ is an analytic function in $V$ with values in $\mathbf{C}^{*}$, and $P_{t}$ is a monic polynomial of degree $k$ ( $k$ being the multiplicity of the function $\xi \mapsto f(0, \ldots, 0, \xi)$ at 0$)$ which depends analytically on $t$ and whose roots $z^{j}(t)$ are in $D(0, \varepsilon)$ for any $t \in \Omega$.

Now consider a compact set $K=K_{1} \times K_{2}$ with $K_{1}$ and $K_{2}$ are compact sets in $\Omega$ and $D(0, \varepsilon)$ respectively.

Since

$$
\frac{1}{f(t, z)} \frac{\partial f(t, z)}{\partial z}=\frac{1}{I(t, z)} \frac{\partial I(t, z)}{\partial z}+\frac{1}{P_{t}(z)} \frac{\partial P_{t}(z)}{\partial z}
$$

and as the meromorphic form $d^{\prime} I / I$ has no singularity in the open set $\Omega \times D(0, \varepsilon)$, it is enough to prove that, for each $j \in\{1, \ldots, p\}$, the integral

$$
\int_{K} \frac{1}{\left|z-z^{j}(t)\right|}\left(\frac{i}{2}\right)^{n} d z_{1} \wedge d \bar{z}_{1} \wedge \ldots \wedge d z_{n-1} \wedge d \bar{z}_{n-1} \wedge d z \wedge d \bar{z}
$$

is finite because

$$
\frac{1}{P_{t}(z)} \frac{\partial P_{t}(z)}{\partial z}=\sum_{j=1}^{k} \frac{1}{z-z^{j}(t)}
$$

For this purpose, take a number $r \in] \varepsilon / 3,2 \varepsilon / 3[$, a point $a \in D(0, r)$, and let us prove that the integral $J(a):=\int_{D(0, \varepsilon)} \frac{1}{|z-a|}\left(\frac{i}{2}\right) d z \wedge d \bar{z}$ is uniformly bounded with respect to $a$. Indeed,

$$
J(a)=\int_{D(0, \varepsilon) \backslash D(a, r / 2)} \frac{1}{|z-a|}\left(\frac{i}{2}\right) d z \wedge d \bar{z}+\int_{D(a, r / 2)} \frac{1}{|z-a|}\left(\frac{i}{2}\right) d z \wedge d \bar{z}
$$


$\leq \frac{2}{r} \int_{D(0, \varepsilon)}\left(\frac{i}{2}\right) d z \wedge d \bar{z}+\int_{D(0, r / 2)} \frac{1}{|z|}\left(\frac{i}{2}\right) d z \wedge d \bar{z}$

$\leq 8 \pi \varepsilon$.

So, by restricting the compact set $K$ if necessary, we can assume that for any $t \in K_{1}$, the roots $z^{j}(t)(1 \leq j \leq k)$ belong to $D(0, r)$. By Fubini's theorem, we have

$$
\int_{K} \frac{1}{\left|z-z^{j}(t)\right|}\left(\frac{i}{2}\right)^{n} d z_{1} \wedge d \bar{z}_{1} \wedge \ldots \wedge d z_{n-1} \wedge d \bar{z}_{n-1} \wedge d z \wedge d \bar{z} \leq 8 \pi \varepsilon \operatorname{mes}\left(K_{1}\right) .
$$

This completes the proof of the existence of the current $\left[d^{\prime} f / f\right]$ when $f$ is any holomorphic nonzero function on $V$.

\section{$\S 4$. Proof of $(L P)$ for "convenient"differential forms.}

In this section, we prove the formula $(L P)$ for differential forms which are locally given by $\varphi=\rho(t, z) d t \wedge d \bar{t}$ where $\rho$ is a smooth function with compact support in $V$, and $d t \wedge d \bar{t}=d z_{1} \wedge \ldots d z_{n-1} \wedge d \bar{z}_{1} \wedge \ldots \wedge d \bar{z}_{n-1}$. We will say that a such $\varphi$ is "convenient" with respect to the projection $\Omega \times D(0, \varepsilon) \rightarrow \Omega,(t, z) \mapsto t$.

First, we consider the case where the function $f$ is such that, for $j \neq j^{\prime}$, the roots $z^{j}$ and $z^{j^{\prime}}$ are different at the generic point $t$ of $\Omega$.

By (1), and since the differential form $d^{\prime} I / I$ is holomorphic on the open set $\Omega \times D(0, \varepsilon)$, we have

$$
d^{\prime \prime}\left[\frac{d^{\prime} f}{f}\right]=d^{\prime \prime}\left[\frac{d^{\prime} P_{t}}{P_{t}}\right]
$$

whence

$$
\begin{gathered}
d^{\prime \prime}\left[\frac{d^{\prime} f}{f}\right](\varphi)=\int_{\Omega \times D(0, \varepsilon)} \frac{d^{\prime} P_{t}}{P_{t}} \wedge d^{\prime \prime} \varphi \\
=\int_{\Omega \times D(0, \varepsilon)}\left(\sum_{j=1}^{n-1} \frac{1}{P_{t}(z)} \frac{\partial P_{t}(z)}{\partial z_{j}} d z_{j}+\frac{P_{t}^{\prime}(z)}{P_{t}(z)} d z\right) \wedge \frac{\partial \rho(t, z)}{\partial \bar{z}} d \bar{z} \wedge d t \wedge d \bar{t} \\
=\int_{\Omega \times D(0, \varepsilon)} \frac{P_{t}^{\prime}(z)}{P_{t}(z)} d z \wedge \frac{\partial \rho(t, z)}{\partial \bar{z}} d \bar{z} \wedge d t \wedge d \bar{t}
\end{gathered}
$$


$=\int_{\Omega} d t \wedge d \bar{t} \int_{D(0, \varepsilon)} \frac{P_{t}^{\prime}(z)}{P_{t}(z)} d z \wedge \frac{\partial \rho(t, z)}{\partial \bar{z}} d \bar{z}$.

By the case $n=1$ and Fubini's theorem, we get

$$
\int_{D(0, \varepsilon)} \frac{1}{2 i \pi} \frac{P_{t}^{\prime}(z)}{P_{t}(z)} d z \wedge \frac{\partial \rho(t, z)}{\partial \bar{z}} d \bar{z}=\left[P_{t}=0\right](\rho(t, \cdot)),
$$

and hence

$$
d^{\prime \prime}\left[\frac{1}{2 i \pi} \frac{d^{\prime} P_{t}}{P_{t}}\right](\varphi)=\int_{\Omega}\left(\sum_{j=1}^{p} \rho\left(t, z^{j}(t)\right)\right) d t \wedge d \bar{t} .
$$

Now consider

$$
R:=\left\{t \in \Omega, \prod_{1 \leq j<j^{\prime} \leq n}\left(z^{j}(t)-z^{j^{\prime}}(t)\right)^{2}=0\right\} .
$$

By the hypothesis on the roots $z^{j}$, we know (see [5]) that $R$ is a closed analytic set with empty interior, hence of Lebesgue measure equal to zero in $\Omega$.

Put $\{f=0\}=\left\{(t, z) \in \Omega \times D(0, \varepsilon), \quad P_{t}(z)=0\right\}$. The local parametrization theorem for analytic sets exhibits the hypersurface $\{f=$ $0\}$ as a branched covering of degree $k$ of $\Omega$ via the natural projection

$$
\pi_{0}:\{f=0\} \rightarrow \Omega,(t, z) \mapsto t,
$$

and the branching locus is $R$. Then we have

$$
\begin{aligned}
& \int_{\Omega}\left(\sum_{j=1}^{k} \rho\left(t, z^{j}(t)\right)\right) d t \wedge d \bar{t}=\int_{\Omega \backslash R}\left(\sum_{j=1}^{k} \rho\left(t, z^{j}(t)\right)\right) d t \wedge d \bar{t} \\
= & \int_{\{f=0\} \backslash \pi_{0}^{-1}(R)} \varphi .
\end{aligned}
$$

Let $S$ be the singular locus of $\{f=0\}$ and put $S_{\varepsilon}=\{x \in\{f=$ $0\}, d(x, S) \leq \varepsilon\}$ with $\varepsilon>0$.

The Lelong theorem (see [6]) gives

$$
\int_{\{f=0\}} \varphi=\lim _{\varepsilon \rightarrow 0} \int_{\{f=0\} \backslash S_{\varepsilon}} \varphi .
$$


Since $\pi_{0}^{-1}(R)$ is a closed analytic space, it is of measure 0 in $\{f=0\}$, then $\pi_{0}^{-1}(R) \cap\left(\{f=0\} \backslash S_{\varepsilon}\right)$ is of measure 0 in the analytic complex manifold $\{f=0\} \backslash S_{\varepsilon}$.

Let $\chi_{\varepsilon}$ be the characteristic function of $\{f=0\} \backslash S_{\varepsilon}$ in the complex analytic manifold $\{f=0\} \backslash \pi_{0}^{-1}(R)$. We have

$$
\int_{\{f=0\} \backslash \pi_{0}^{-1}(R)} \chi_{\varepsilon} \varphi=\int_{\{f=0\} \backslash S_{\varepsilon}} \varphi
$$

which implies

$$
\lim _{\varepsilon \rightarrow 0} \int_{\{f=0\} \backslash \pi_{0}^{-1}(R)} \chi_{\varepsilon} \varphi=\int_{\{f=0\}} \varphi
$$

Moreover

$$
\sum_{j=1}^{k}\left|\chi_{\varepsilon} \rho\left(t, z^{j}(t)\right)\right| \leq \sum_{j=1}^{k}\left|\rho\left(t, z^{j}(t)\right)\right|
$$

where the term on the right is independant on $\varepsilon$ and integrable since

$$
\rho\left(t, z^{j}(t)\right) d t \wedge d \bar{t}=\left(\pi_{0}\right)_{*} \varphi
$$

and since the differential form $\varphi$ has continuous coefficients on $V$ (see [4]) and has a compact support.

As $\left(\chi_{\varepsilon} \varphi\right)_{\varepsilon}$ converges almost everywhere to $\varphi$ when $\varepsilon$ tends to 0 , the Lebesgue's dominated convergence theorem gives

$$
\lim _{\varepsilon \rightarrow 0} \int_{\{f=0\} \backslash \pi_{0}^{-1}(R)} \chi_{\varepsilon} \varphi=\int_{\{f=0\} \backslash \pi_{0}^{-1}(R)} \varphi .
$$

Then we have

$$
\int_{\{f=0\}} \varphi=\int_{\{f=0\} \backslash \pi_{0}^{-1}(R)} \varphi=d^{\prime \prime}\left[\frac{1}{2 i \pi} \frac{d^{\prime} f}{f}\right](\varphi),
$$


which establishes the formula $(L P)$ when the branching locus does not coincide with $\Omega$.

Now we are ready to prove $(L P)$ for any holomorphic function on $V$.

We keep the notations of $\S 1$. We know that the vanishing locus of $f$ in $\Omega \times D(0, \varepsilon)$ consists in that of the function $(t, z) \mapsto P(t, z)$ which is the branching covering of degree $k$ over the open set $\Omega$. This covering can be seen as an analytic application $P: \Omega \rightarrow \mathbf{C}^{k}$ where $\mathbf{C}^{k}$ is identified here to the set of monic polynomials of degree $k$ with complex coefficients.

We call the ring of functions of $P$, and we denote by $\mathcal{O}(P)$, the quotient of the ring $\mathcal{O}(\Omega \times D(0, \varepsilon))$ of holomorphic functions on $\Omega \times D(0, \varepsilon)$, by the principal ideal generated by $\widetilde{P}: \Omega \times D(0, \varepsilon) \rightarrow \mathbf{C},(t, z) \mapsto \widetilde{P}(t, z):=$ $P_{t}(z)$.

The branched covering $P$ is said to be reduced if the ring $\mathcal{O}(P)$ is reduced, that is, every nilpotent element in $\mathcal{O}(P)$ is zero. $P$ is said to be irreducible if $\mathcal{O}(P)$ is integral.

Since $P$ is reduced if and only if its branching locus $R$ does not coincide with $\Omega$ (see [3]), we deduce that the formula $(L P)$ has been proved when $P$ is reduced. To get this result with any $f$, it is enough now to use the decomposition theorem (see [3]) which asserts that the branching covering $P$ induced by $f$ can be decomposed in a unique way in the form $P=$ $\prod_{j} P_{j}^{n_{j}}$ where $P_{j}$ are reduced (and irreducible) branching coverings, and $n_{j}$ are positive integers.

Indeed, if $f$ is any holomorphic function on the open set $V=\Omega \times D(0, \varepsilon)$, then the decomposition theorem allows to write $f=\prod_{j} f_{j}^{n_{j}}$ where the $f_{j}$ are reduced and irreducible. From the result proved in $\S 4$ we deduce

$$
d^{\prime \prime}\left[\frac{1}{2 i \pi} \frac{d^{\prime} f}{f}\right]=\sum_{j} n_{j} d^{\prime \prime}\left[\frac{1}{2 i \pi} \frac{d^{\prime} f_{j}}{f_{j}}\right]=\sum_{j} n_{j}\left[f_{j}=0\right]=[f=0] .
$$

The proof of $(L P)$ is then complete for any $(n-1, n-1)$ - form in $\mathcal{D}(V)$ locally given by $\varphi(t, z))=\rho(t, z) d t \wedge d \bar{t}$. 
It remains to show that the result above is still true for any compactly supported $(n-1, n-1)$ - form of class $C^{\infty}$ on $V$. It is the aim of the following section.

\section{$\S 3$. Proof of $(L P)$ in the general case.}

We want to prove the equality

$$
d^{\prime \prime}\left[\frac{1}{2 i \pi} \frac{d^{\prime} f}{f}\right](\varphi)=\int_{\{f=0\}} \varphi
$$

for any $(n-1, n-1)$ - form $\varphi$ in $\mathcal{D}(V)$.

By the local parametrization theorem, we may assume that

- $V=\Omega \times \mathbf{C}$ where $\Omega$ is a domain $\mathbf{C}^{n-1}$,

- $\{f=0\}$ is a branching covering of degree $k$ over $\Omega$ via the projection $\pi_{0}: V \rightarrow \Omega$.

- there exists a compact set $K$ in $\Omega$ such that the support of $\varphi$ is contained in $K \times \mathbf{C}$.

Then, there exists a neighborhood $U$ of 0 in $L\left(\mathbf{C}^{n}, \mathbf{C}^{n-1}\right)$ such that for every $u \in U$, the projection $\pi_{u}:=\pi_{0}+u$ exhibits $\Omega$ as a branching covering of a same neighborhood $\Omega^{\prime}$ of $K$ in $\Omega$ (more precisely, such that $\pi_{u}^{-1}\left(\Omega^{\prime}\right) \cap\{f=0\} \rightarrow \Omega^{\prime}$ is a branching covering of degree $k$ ).

The following lemma shows that it is sufficient to consider "sympathic" forms with respect to the given projection.

Lemma Let $\pi_{0}: \mathbf{C}^{n+p} \rightarrow \mathbf{C}^{n}$ be the canonical projection. For $u \in$ $L\left(\mathbf{C}^{n+p}, \mathbf{C}^{n}\right)$, set $\pi_{u}=\pi_{0}+u$. For any couple of integers $(a, b)$ such that $a \leq n$ et $b \leq n$, and for any neighborhood $U$ of 0 in $L\left(\mathbf{C}^{n+p}, \mathbf{C}^{n}\right)$, we have

$$
\Lambda^{a, b}\left(\mathbf{C}^{n+p}\right)^{*}=\sum_{u \in U} \pi_{u}^{*}\left(\Lambda^{a, b}\left(\mathbf{C}^{n}\right)^{*}\right)
$$

where $\Lambda^{a, b}(E)^{*}$, for a complex vector space $E$, denotes the space of $a$-linear and $b$-antilinear alternating forms on $E$. 
By duality and analytic extension with respect to $u$, this lemma is an immediate consequence of the following result.

Proposition Let $n \in \mathbf{N}^{*}$ and $p \in \mathbf{N}$. Let $a$ and $b$ be integers such that $a \leq n$ and $b \leq n$, and let $v \in \Lambda^{a, b}\left(\mathbf{C}^{n+p}\right)^{*}$. If for any $u \in L\left(\mathbf{C}^{n+p}, \mathbf{C}^{n}\right)$ we have $u_{*}(v)=0$, then $v=0$.

Proof Following [2], we establish this result by induction on $p$.

For $p=0$, the result is obvious. Then we may assume $p \geq 1$.

Setting $\mathbf{C}^{n+p}=H \oplus \mathbf{C} e$, we have

$\Lambda^{a, b}\left(\mathbf{C}^{n+p}\right)=\Lambda^{a, b}(H) \oplus \Lambda^{a-1, b}(H) \wedge e \oplus \Lambda^{a, b-1}(H) \wedge \bar{e} \oplus \Lambda^{a-1, b-1}(H) \wedge e \wedge \bar{e}$.

Let $v=v_{0,0} \oplus v_{1,0} \wedge e \oplus v_{0,1} \wedge \bar{e} \oplus v_{1,1} \wedge e \wedge \bar{e}$.

- If $v_{0,0} \neq 0$, then, by induction hypothesis, there exists $f \in L\left(H, \mathbf{C}^{n}\right)$ such that $f_{*}\left(v_{0,0}\right) \neq 0$. Putting $u=f$ on $H$ and $u(e)=0$, we define an element of $L\left(C^{n+p}, \mathbf{C}^{n}\right)$ which satisfies $u_{*}(v)=u_{*}\left(v_{0,0}\right)=f_{*}\left(v_{0,0}\right) \neq 0$. This establishes the result in this case.

- If $v_{1,1} \neq 0$, then, by induction hypothesis (because $a-1 \leq n-1$ and $b-1 \leq n-1)$, there exists $g \in L\left(H, \mathbf{C}^{n-1}\right)$ such that $g_{*}\left(v_{1,1}\right) \neq 0$. Put $\mathbf{C}^{n}=\mathbf{C}^{n-1} \oplus \mathbf{C} \varepsilon$, and define $u$ in $L\left(\mathbf{C}^{n+p}, \mathbf{C}^{n}\right)$ by $u=g \oplus 0$ on $H$ and $u(e)=\varepsilon$. Then the component on $\Lambda^{a-1, b-1}\left(\mathbf{C}^{n-1}\right) \wedge \varepsilon \wedge \bar{\varepsilon}$ of $u_{*}(v)$ is $g_{*}\left(v_{1,1}\right) \wedge \varepsilon \wedge \bar{\varepsilon} \neq 0$, wich completes this case.

- Assume now that $v_{0,0}=v_{1,1}=0$ and $v_{1,0} \neq 0$ (for instance). Let $w$ be a totally decomposed vector in $\Lambda^{a-1, b}(H)^{*}$ such that $\left\langle v_{1,0}, w\right\rangle=1$, and put

$$
w=w_{1} \wedge \ldots \wedge w_{a-1} \wedge \overline{t_{1}} \wedge \ldots \overline{t_{b}},
$$

where the $w_{i}$ and the $t_{j}$ are in $H^{*}$. Since $a-1 \leq n-1<n+p-1=$ $\operatorname{dim}_{\mathbf{C}} H$, there exists a nonzero element in $\bigcap_{i=1}^{n-1} \operatorname{Ker} w_{i}$.

Let $h^{*}$ be an element of $H^{*}$ such that $\left\langle h^{*}, h\right\rangle=1$. Then

$$
\left\langle v_{1,0} \wedge h, w \wedge h^{*}\right\rangle=\left\langle v_{1,0}, w\right\rangle=1 .
$$


We deduce that $v_{1,0} \wedge h$ is nonzero in $\Lambda^{a, b}(H)$. By the induction hypothesis there exists $f \in L\left(H, \mathbf{C}^{n}\right)$ such that $f_{*}\left(v_{1,0} \wedge h\right) \neq 0$.

Consider now the linear application $g: \mathbf{C}^{n+p} \rightarrow H$ defined by $g_{\mid H}=$ $\operatorname{Id}_{H}$ and $g(e)=h$. Then, for $u=f \circ g$ we get $u_{*}\left(v_{1,0} \wedge e\right) \neq 0$. Moreover, if $h^{\prime}$ is close to $h$ in $H$, and if $h^{\prime}$ is close to $f$ in $L\left(H, \mathbf{C}^{n}\right)$, this property will remain true. We deduce

$$
f^{\prime}\left(v_{1,0}\right) \wedge f^{\prime}\left(h^{\prime}\right)+f^{\prime}\left(v_{0,1}\right) \wedge \overline{f^{\prime}\left(h^{\prime}\right)}=0
$$

for at least one $f^{\prime}$ which can be assumed to be of rank $n$ and for any $h^{\prime}$ close to $h$. Since $f^{\prime}$ is of maximum rank, $f^{\prime}\left(h^{\prime}\right)$ will describe a neighborhood of $f^{\prime}(h)$ when $h^{\prime}$ describes a neighborhood of $h$ in $H$. Thus, we get the desired contradiction. Indeed, if $A$ and $B$ are elements of $\Lambda^{a-1, b}\left(\mathbf{C}^{n}\right)$ and $\Lambda^{a, b-1}\left(\mathbf{C}^{n}\right)$ respectively, and if $A \wedge v+B \wedge \bar{v}=0$ for any $v$ in an open subset of $\mathbf{C}^{n}$, then $A=B=0$.

This completes the proof of the proposition.

Remark : In the previous lemma, it is clear that, for a given $U$, it is sufficient to consider a finite number of $u$ in $U$.

Example : For $n=2$ and $\lambda \in \mathbf{C}$, consider the mappings

$$
\pi_{\lambda}: \mathbf{C}^{2} \rightarrow \mathbf{C}, \quad\left(z_{1}, z_{2}\right) \mapsto z_{1}+\lambda z_{2} .
$$

We have

$\pi_{\lambda}^{*}\left(d z_{1} \wedge d \bar{z}_{1}\right)=d z_{1} \wedge d \bar{z}_{1}+\bar{\lambda} d z_{1} \wedge d \bar{z}_{2}+\lambda d z_{2} \wedge d \bar{z}_{1}+\lambda \bar{\lambda} d z_{2} \wedge d \bar{z}_{2}$,

and for $\lambda$ such that $\lambda \neq \pm \bar{\lambda}$, the family

$$
\left\{\pi_{\lambda}^{*}\left(d z_{1} \wedge d \bar{z}_{1}\right), \pi_{-\lambda / 2}^{*}\left(d z_{1} \wedge d \bar{z}_{1}\right), \pi_{\bar{\lambda}}^{*}\left(d z_{1} \wedge d \bar{z}_{1}\right), \pi_{\bar{\lambda} / 4}^{*}\left(d z_{1} \wedge d \bar{z}_{1}\right)\right\}
$$

is free, and then generates $\Lambda^{1,1}\left(\mathbf{C}^{2}\right)^{*}$. 
By the lemma, and up to a finite number of projections closed to $\pi_{0}$, it is sufficient to consider the case $\varphi(t, z)=\rho(t, z) d t \wedge \bar{t}$ where $t_{1}, \ldots, t_{n-1}$ and $z$ are coordinates on $\mathbf{C}^{n-1}$ and $\mathbf{C}$ respectively, $d t \wedge d \bar{t}=d t_{1} \wedge \ldots d t_{n-1} \wedge$ $d \bar{t}_{1} \wedge \ldots \wedge d \bar{t}_{n-1}$, and $\rho$ is a smooth function on $\Omega \times \mathbf{C}$ with compact support in $K \times \mathbf{C}$.

Remark Since $\log |f|$ is plurisubharmonic on $V$, it is locally integrable (see $[7])$. Then it defines a $(0,0)$ - current on $V$.

Introducing the real operator

$$
d^{c}=\frac{d^{\prime}-d^{\prime \prime}}{2 i \pi}
$$

we have $d d^{c}=\frac{i}{\pi} d^{\prime} d^{\prime \prime}$, and then we get

$$
d d^{c} \log |f|=[f=0]
$$

which is nothing but the usual Lelong-Poincaré formula.

\section{References}

[1] Barlet D. : Le théorème d'intégration sur un ensemble analytique complexe de P. Lelong. Séminaire de géométrie analytique. Institut Elie Cartan 5, pp. 1-11, (1982).

[2] Barlet D. : Espace analytique réduit des cycles analytiques complexes compacts d'un espace analytique complexe de dimension finie. Fonctions de plusieurs variables complexes II. Lecture Notes in Mathematics 482, pp. 1-159.

[3] Barlet D. : Convexité de l'espace des cycles. Bull. Soc. Math. France 106, pp. 373-397, (1978).

[4] Demailly J.-P. : Courants positifs et théorie de l'intersection. Gaz. Math. 53, pp. 131-159, (1992). 
[5] Gunning P., Rossi H. : Analytic functions of several complex variables. AMS Chelsea Publishing, Providence, RI, (2009).

[6] Lelong P. : Intégration sur un ensemble analytique complexe. Bull. Soc. Math. France, 85, pp. 239-262, (1957).

[7] Lelong P. : Fonctions plurisousharmoniques et formes différentielles positives. Dunod, Paris, Gordon \& Breach, New-York, (1968).

\author{
M. El Amrani \\ Université d'Angers \\ Département de Mathématiques. \\ U.F.R. Sciences. 2, \\ Boulevard Lavoisier. 49045 Angers Cedex 01. \\ France \\ e-mail : mohammed.elamrani@univ-angers.fr \\ and
}

\author{
A. Jeddi \\ Institut Elie Cartan, \\ Université (et Archives de) H. Poincaré, \\ BP 239, \\ Vandoeuvre-les-Nancy Cedex. \\ France \\ e-mail : ahmed.jeddi@iecn.u-nancy.fr
}

